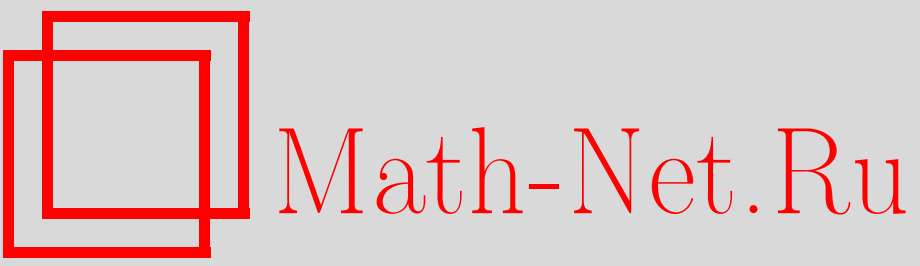

М. М. Кобилзода, А. Н. Наимов, Об ограниченных решениях одного класса систем обыкновенных дифференциальных уравнений, Итоги науки и техн. Сер. Соврем. мат. и ее прил. Темат. обз., 2021, том 192, 65-73

DOI: https://doi.org/10.36535/0233-6723-2021-192-65-73

Использование Общероссийского математического портала Math-Net.Ru подразумевает, что вы прочитали и согласны с пользовательским соглашением

http://www.mathnet.ru/rus/agreement

Параметры загрузки:

IP : 54.84 .234 .179

26 апреля 2023 г., 16:14:49 


\title{
ОБ ОГРАНИЧЕННЫХ РЕШЕНИЯХ ОДНОГО КЛАССА СИСТЕМ ОБЫКНОВЕННЫХ ДИФФЕРЕНЦИАЛЬНЫХ УРАВНЕНИЙ
}

\author{
(c) 2021 г. $\quad$ М. М. КОБИЛЗОДА, А. Н. НАИМОВ
}

\begin{abstract}
АннотАция. В статье исследован вопрос об априорной оценке и существовании ограниченных решений для класса систем обыкновенных дифференциальных уравнений, правые части которых могут иметь произвольный порядок роста по независимой и зависимым переменным. Найдены односторонние оценка правых части уравнений, обеспечивающие априорную оценку ограниченных решений и при помощи методов вычисления вращения векторных полей и метода периодических срезок доказаны теоремы о существовании периодических и ограниченных решений.
\end{abstract}

Ключевъе слова: односторонняя оценка, ограниченное решение, периодическое решение, априорная оценка, вращение векторного поля.

\section{ON BOUNDED SOLUTIONS OF ONE CLASS OF SYSTEMS OF ORDINARY DIFFERENTIAL EQUATIONS}

\author{
(c) 2021 M. M. KOBILZODA, A. N. NAIMOV
}

\begin{abstract}
In this paper, we consider a priori estimates and examine the existence of bounded solutions for a class of systems of ordinary differential equations whose right-hand sides have an arbitrary order of growth with respect to the independent and dependent variables. For the right-hand sides of the equations, we find one-sided estimates that provide a priori estimates for bounded solutions; using the methods for calculating the rotation of vector fields and the method of periodic cuts, we are prove theorems on the existence of periodic and bounded solutions.
\end{abstract}

Keywords and phrases: one-sided estimate, bounded solution, periodic solution, ä̈priori estimate, rotation of a vector field .

AMS Subject Classification: 34B15, 34B40

1. Введение. Статья посвящена исследованию ограниченных решений систем обыкновенных дифференциальных уравнений вида

$$
v_{k}^{\prime}=F_{k}\left(t, v_{1}, v_{2}\right), \quad v_{k} \in \mathbb{C}^{n_{k}}, \quad t \in \mathbb{R}, \quad k=1,2,
$$

где $\mathbb{R}=(-\infty,+\infty)$ - вещественная ось, $\mathbb{C}^{n_{k}}$ - пространство $n_{k}$-мерных комплексных векторов, $F_{k}: \mathbb{R} \times \mathbb{C}^{n_{1}} \times \mathbb{C}^{n_{2}} \mapsto \mathbb{C}^{n_{k}}, k=1,2$ - заданные непрерывные отображения.

Ограниченным решением системы уравнений (1) называем пару $\left(v_{1}(t), v_{2}(t)\right)$ вектор-функций $v_{k}: \mathbb{R} \mapsto \mathbb{C}^{n_{k}}, k=1,2$, ограниченных, непрерывно дифференцируемых и удовлетворяющих уравнениям системы (1).

Ограниченные решения систем обыкновенных дифференциальных уравнений исследованы в многочисленных работах. Отметим работы [1,3-5], где исследованы вопросы априорной оценки и существования ограниченных решений, применяя идеи и методы линейного и нелинейного

Работа выполнена при поддержке Российского фонда фундаментальных исследований (проекты № 18-47-350001 p-a, № 19-01-00103a). 
функционального анализа. В [3,5] рассмотрены системы линейных обыкновенных дифференциальных уравнений с переменными и ограниченными коэффициентами, а в $[1,4]$ - системы нелинейных обыкновенных дифференциальных уравнений. В отличие от указанных работ в исследуемой системе уравнений (1) отображения $F_{k}\left(t, v_{1}, v_{2}\right), k=1,2$, могут иметь любой порядок роста относительно $t$ и $v_{1}, v_{2}$ при $|t| \rightarrow+\infty$ и $\left|v_{1}\right|+\left|v_{2}\right| \rightarrow+\infty$.

В настоящей работе найдены новые условия в виде односторонних оценок, при выполнении которых имеет место априорная оценка для ограниченных решений системы уравнений (1). В условиях априорной оценки сперва доказано существование $\omega$-периодического решения, предполагая правые части $\omega$-периодическими по $t$ и применяя методы вычисления вращения векторных полей [2]. А затем доказано существование ограниченного решения, применяя метод периодических срезок $[1,4]$. Полученные результаты по аналогии с работами $[6,7]$ можно применять при исследовании ограниченных решений новых классов систем линейных и нелинейных дифференциальных уравнений в частных производных.

2. Основные результаты. Введем банаховы пространства $E^{k}, k=1,2$ непрерывных и ограниченных вектор-функций $w_{k}: \mathbb{R} \mapsto \mathbb{C}^{n_{k}}$ с нормами

$$
\left\|w_{k}\right\|_{k}:=\sup _{t \in \mathbb{R}}\left|w_{k}(t)\right|_{k}, \quad k=1,2,
$$

где $|f|_{k}^{2}=\langle f, f\rangle_{k},\langle f, g\rangle_{k}=f_{1} \overline{g_{1}}+\ldots+f_{n_{k}} \overline{g_{n_{k}}}$ - скалярное произведение в $\mathbb{C}^{n_{k}}$.

Существование ограниченного решения системы уравнений (1) исследуем в условиях априорной оценки. Скажем, что для ограниченных решений системы уравнений (1) имеет место априорная оценка, если множество ограниченных решений системы уравнений (1) либо пусто, либо ограничено по норме

пространства $E^{1} \times E^{2}$.

$$
\left\|\left(w_{1}, w_{2}\right)\right\|_{E^{1} \times E^{2}}:=\sqrt{\left\|w_{1}\right\|_{1}^{2}+\left\|w_{2}\right\|_{2}^{2}}
$$

Предположим, что существуют вещественные числа $\alpha_{k}>0, \beta_{k} \geqslant 0, \gamma_{k} \geqslant 0, k=1,2$, такие, что отображения $F_{1}\left(t, z_{1}, z_{2}\right)$ и $F_{2}\left(t, z_{1}, z_{2}\right)$ при всех $t \in \mathbb{R}, z_{1} \in \mathbb{C}^{n_{1}}, z_{2} \in \mathbb{C}^{n_{2}}$ удовлетворяют условиям

$$
\begin{aligned}
& \operatorname{Re}\left\langle F_{1}\left(t, z_{1}, z_{2}\right), z_{1}\right\rangle_{1} \leqslant-\alpha_{1}\left|z_{1}\right|_{1}^{2}+\beta_{1}\left|z_{2}\right|_{2}^{2}+\gamma_{1}, \\
& \operatorname{Re}\left\langle F_{2}\left(t, z_{1}, z_{2}\right), z_{2}\right\rangle_{2} \geqslant \alpha_{2}\left|z_{2}\right|_{2}^{2}-\beta_{2}\left|z_{1}\right|_{1}^{2}-\gamma_{2} .
\end{aligned}
$$

В условиях односторонних оценок (2) и (3) отображения $F_{1}\left(t, z_{1}, z_{2}\right)$ и $F_{2}\left(t, z_{1}, z_{2}\right)$ могут иметь любой порядок роста по $t$ и $z_{1}, z_{2}$ при $|t| \rightarrow+\infty$ и $\left|z_{1}\right|_{1}+\left|z_{2}\right|_{2} \rightarrow+\infty$. Имеет место следующая теорема об априорной оценке ограниченных решений.

Теорема 1. Пусть выполнены условия (2), (3) и условие

$$
\beta_{1} \beta_{2}<\alpha_{1} \alpha_{2} \text {. }
$$

Тогда для любого ограниченного решения системы уравнений (1) верна оценка

$$
\left\|v_{1}\right\|_{1}^{2}+\left\|v_{2}\right\|_{2}^{2} \leqslant M
$$

где

$$
M=\left(1-\frac{\beta_{1}}{\alpha_{1}} \frac{\beta_{2}}{\alpha_{2}}\right)^{-1}\left(\frac{\gamma_{1}}{\alpha_{1}}\left(1+\frac{\beta_{2}}{\alpha_{2}}\right)+\frac{\gamma_{2}}{\alpha_{2}}\left(1+\frac{\beta_{1}}{\alpha_{1}}\right)\right) .
$$

В частном случае, когда отображения $F_{1}\left(t, z_{1}, z_{2}\right)$ и $F_{2}\left(t, z_{1}, z_{2}\right)$ периодичны по $t$ с периодом $\omega>0$, имеет место априорная оценка (5) для всех $\omega$-периодических решений, при этом число $M$ не зависит от величины периода $\omega$. Справедлива следующая теорема.

Теорема 2. Если выполнены условия (2)-(4) и отображения $F_{1}\left(t, z_{1}, z_{2}\right), F_{2}\left(t, z_{1}, z_{2}\right)$ периодичны по $t$ с периодом $\omega$, то существует $\omega$-периодическое решение системы уравнений (1).

Теорема 2 доказана с применением методов вычисления вращения векторных полей [2]. При гомотопии и вычислении вращения векторного поля, порожденного $\omega$-периодической задачей, существенно используются односторонние оценки (2) и (3).

Из приведенных выше теорем вытекает следующее утверждение. 
Теорема 3. Пусть выполнены условия (2)-(4). Тогда существует хотя бы одно ограниченное решение системы уравнений (1).

Теорема 3 доказана методом периодических срезок (см. $[1,4])$.

3. Априорная оценка ограниченных решений. Приведем доказательство теоремы 1 . Пусть $\left(v_{1}(t), v_{2}(t)\right)$ - ограниченное решение системы уравнений (1). Уравнения системы (1) скалярно перемножая с $v_{k}(t), k=1,2$, получаем равенства:

$$
\left(\left|v_{k}(t)\right|_{k}^{2}\right)^{\prime}=2 \operatorname{Re}\left\langle F_{k}\left(t, v_{1}(t), v_{2}(t)\right), v_{k}(t)\right\rangle_{k}, \quad t \in \mathbb{R}, \quad k=1,2 .
$$

Эти равенства перепишем в следующем виде:

$$
\left(\left|v_{k}(t)\right|_{k}^{2}\right)^{\prime}+(-1)^{k-1} 2 \alpha_{k}\left|v_{k}(t)\right|_{k}^{2}=g_{k}(t), \quad t \in \mathbb{R}, \quad k=1,2,
$$

где

$$
g_{k}(t)=(-1)^{k-1} 2 \alpha_{k}\left|v_{k}(t)\right|_{k}^{2}+2 \operatorname{Re}\left\langle F_{k}\left(t, v_{1}(t), v_{2}(t)\right), v_{k}(t)\right\rangle_{k} .
$$

Решим дифференциальные уравнения (6) относительно функций $\left|v_{k}(t)\right|_{k}^{2}, k=1,2$ :

$$
\left|v_{k}(t)\right|_{k}^{2}=e^{(-1)^{k} 2 \alpha_{k} t}\left(\left|v_{k}(0)\right|_{k}^{2}+\int_{0}^{t} e^{-(-1)^{k} 2 \alpha_{k} s} g_{k}(s) d s\right), \quad k=1,2 .
$$

Отсюда в силу ограниченности функций $\left|v_{k}(t)\right|_{k}^{2}, k=1,2$, следует существование пределов

$$
\lim _{(-1)^{k} t \rightarrow+\infty}\left(\left|v_{k}(0)\right|_{k}^{2}+\int_{0}^{t} e^{-(-1)^{k} 2 \alpha_{k} s} g_{k}(s) d s\right)=\lim _{(-1)^{k} t \rightarrow+\infty}\left|v_{k}(t)\right|_{k}^{2} e^{-(-1)^{k} 2 \alpha_{k} t}=0, \quad k=1,2 .
$$

Следовательно, несобственные интегралы

$$
\int_{-\infty}^{0} e^{2 \alpha_{1} s} g_{1}(s) d s, \quad \int_{0}^{+\infty} e^{-2 \alpha_{2} s} g_{2}(s) d s
$$

сходятся, и имеют место равенства

$$
\left|v_{1}(0)\right|_{1}^{2}=\int_{-\infty}^{0} e^{2 \alpha_{1} s} g_{1}(s) d s, \quad\left|v_{2}(0)\right|_{2}^{2}=-\int_{0}^{+\infty} e^{-2 \alpha_{2} s} g_{2}(s) d s
$$

Подставляя эти значения в формулы общего решения, имеем:

$$
\left|v_{1}(t)\right|_{1}^{2}=\int_{-\infty}^{t} e^{-2 \alpha_{1}(t-s)} g_{1}(s) d s, \quad\left|v_{2}(t)\right|_{2}^{2}=-\int_{t}^{+\infty} e^{-2 \alpha_{2}(s-t)} g_{2}(s) d s
$$

Функции $g_{1}(t)$ и $g_{2}(t)$ в силу условий $(2)$ и $(3)$, удовлетворяют неравенствам

$$
g_{1}(t) \leqslant 2 \beta_{1}\left|v_{2}(t)\right|_{2}^{2}+2 \gamma_{1}, \quad g_{2}(t) \geqslant 2 \beta_{2}\left|v_{1}(t)\right|_{1}^{2}+2 \gamma_{2} .
$$

Учитывая это, из (7) получаем следующие неравенства:

$$
\begin{aligned}
& \left|v_{1}(t)\right|_{1}^{2} \leqslant 2\left(\beta_{1}\left\|v_{2}\right\|_{2}^{2}+\gamma_{1}\right) \int_{-\infty}^{t} e^{-2 \alpha_{1}(t-s)} d s, \\
& \left|v_{2}(t)\right|_{2}^{2} \leqslant 2\left(\beta_{2}\left\|v_{1}\right\|_{1}^{2}+\gamma_{2}\right) \int_{t}^{+\infty} e^{-2 \alpha_{2}(s-t)} d s .
\end{aligned}
$$


Из полученных неравенств выводим:

$$
\begin{array}{cl}
\left\|v_{1}\right\|_{1}^{2} \leqslant \frac{1}{\alpha_{1}}\left(\beta_{1}\left\|v_{2}\right\|_{2}^{2}+\gamma_{1}\right), & \left\|v_{2}\right\|_{2}^{2} \leqslant \frac{1}{\alpha_{2}}\left(\beta_{2}\left\|v_{1}\right\|_{1}^{2}+\gamma_{2}\right), \\
\left\|v_{1}\right\|_{1}^{2} \leqslant \frac{\beta_{1}}{\alpha_{1}}\left(\frac{\beta_{2}}{\alpha_{2}}\left\|v_{1}\right\|_{1}^{2}+\frac{\gamma_{2}}{\alpha_{2}}\right)+\frac{\gamma_{1}}{\alpha_{1}}, & \left\|v_{2}\right\|_{2}^{2} \leqslant \frac{\beta_{2}}{\alpha_{2}}\left(\frac{\beta_{1}}{\alpha_{1}}\left\|v_{2}\right\|_{2}^{2}+\frac{\gamma_{1}}{\alpha_{1}}\right)+\frac{\gamma_{2}}{\alpha_{2}}, \\
\left\|v_{1}\right\|_{1}^{2} \leqslant\left(1-\frac{\beta_{1}}{\alpha_{1}} \frac{\beta_{2}}{\alpha_{2}}\right)^{-1}\left(\frac{\beta_{1}}{\alpha_{1}} \frac{\gamma_{2}}{\alpha_{2}}+\frac{\gamma_{1}}{\alpha_{1}},\right), & \left\|v_{2}\right\|_{2}^{2} \leqslant\left(1-\frac{\beta_{1}}{\alpha_{1}} \frac{\beta_{2}}{\alpha_{2}}\right)^{-1}\left(\frac{\beta_{2}}{\alpha_{2}} \frac{\gamma_{1}}{\alpha_{1}}+\frac{\gamma_{2}}{\alpha_{2}},\right), \\
\left\|v_{1}\right\|_{1}^{2}+\left\|v_{2}\right\|_{2}^{2} \leqslant\left(1-\frac{\beta_{1}}{\alpha_{1}} \frac{\beta_{2}}{\alpha_{2}}\right)^{-1}\left(\frac{\gamma_{1}}{\alpha_{1}}\left(1+\frac{\beta_{2}}{\alpha_{2}}\right)+\frac{\gamma_{2}}{\alpha_{2}}\left(1+\frac{\beta_{1}}{\alpha_{1}}\right)\right) .
\end{array}
$$

Теорема 1 доказана.

4. Существование периодического решения. Для доказательства теоремы 2 рассмотрим семейство вполне непрерывных векторных полей

$$
\Phi_{\lambda}\left(w_{1}, w_{2}\right)=\left(\begin{array}{c}
w_{1}(t)-w_{1}(\omega)-\int_{0}^{t} F_{1 \lambda}\left(s, w_{1}(s), w_{2}(s)\right) d s \\
w_{2}(t)-w_{2}(\omega)-\int_{0}^{t} F_{2 \lambda}\left(s, w_{1}(s), w_{2}(s)\right) d s
\end{array}\right), \quad \lambda \in[0,1],
$$

где

$$
F_{k \lambda}\left(t, z_{1}, z_{2}\right)=(1-\lambda) F_{k}\left(t, z_{1}, z_{2}\right)+\lambda(-1)^{k} \alpha_{k} z_{k}, \quad k=1,2,
$$

в банаховом пространстве $\left.E_{\omega}^{1,2}=C\left([0, \omega] ; \mathbb{C}^{n_{1}}\right) \times \mathbb{C}^{n_{2}}\right)$ с нормой

$$
\left\|\left(w_{1}, w_{2}\right)\right\|^{2}:=\max _{0 \leqslant t \leqslant \omega}\left|w_{1}(t)\right|_{1}^{2}+\max _{0 \leqslant t \leqslant \omega}\left|w_{2}(t)\right|_{2}^{2} .
$$

Легко проверить, что, во-первых, при фиксированном $\lambda \in[0,1]$ пара $\left(w_{1}, w_{2}\right) \in E_{\omega}^{1,2}$ будет $\omega$ периодическим решением системы уравнений

$$
v_{k}^{\prime}=F_{k \lambda}\left(t, v_{1}, v_{2}\right), \quad k=1,2,
$$

лишь в том случае, когда $\Phi_{\lambda}\left(w_{1}, w_{2}\right)=0$, т.е. $\left(w_{1}, w_{2}\right)$ является нулем векторного поля $\Phi_{\lambda}$. Вовторых, при каждом $\lambda \in[0,1]$ отображения $F_{k \lambda}\left(t, z_{1}, z_{2}\right), k=1,2$, удовлетворяют условиям (2) и (3). Отсюда в силу теоремы 1 вытекает, что $\Phi_{\lambda}\left(w_{1}, w_{1}\right) \neq 0$ при любых $\lambda \in[0,1]$ и $\left(w_{1}, w_{2}\right) \in E_{\omega}^{1,2}$, $\left\|\left(w_{1}, w_{2}\right)\right\|>\sqrt{M}$. Следовательно, при любом $\lambda \in[0,1]$ определено вращение $\gamma_{\infty}\left(\Phi_{\lambda}\right)$ векторного поля $\Phi_{\lambda}$ на бесконечности [2] и

$$
\gamma_{\infty}\left(\Phi_{0}\right)=\gamma_{\infty}\left(\Phi_{1}\right)
$$

Покажем, что

$$
\gamma_{\infty}\left(\Phi_{1}\right)=1
$$

Тогда из $(8)$ и $(9)$ следует, что $\gamma_{\infty}\left(\Phi_{0}\right) \neq 0$, и согласно принципу ненулевого вращения (см. [2]) существует хотя бы один нуль векторного поля $\Phi_{0}$. Этим самым теорема 2 будет доказана.

Для доказательства равенства (9) векторное поле $\Phi_{1}$ гомотопируем к конечномерному векторному полю следующей формулой:

$$
\Psi_{\lambda}\left(w_{1}, w_{2}\right)=\left(\begin{array}{c}
w_{1}(t)-w_{1}(\omega)+\lambda \alpha_{1} \int_{0}^{t} w_{1}(s) d s+(1-\lambda) \alpha_{1} \int_{0}^{\omega} w_{1}(s) d s \\
w_{2}(t)-w_{2}(\omega)-\lambda \alpha_{2} \int_{0}^{t} w_{2}(s) d s-(1-\lambda) \alpha_{2} \int_{0}^{\omega} w_{2}(s) d s
\end{array}\right), \quad \lambda \in[0,1] .
$$


Очевидно, $\Psi_{1}=\Phi_{1}$. Проверим, что при любых $\lambda \in[0,1]$ и $\left(w_{1}, w_{1}\right) \in E_{\omega}^{1,2} \backslash\{0,0\}$ имеет место неравенство

$$
\Psi_{\lambda}\left(w_{1}, w_{2}\right) \neq 0
$$

Действительно, если (10) не верно, то при некоторых $\lambda \in[0,1]$ и $\left(w_{1}, w_{1}\right) \in E_{\omega}^{1,2}$ имеем:

$$
w_{k}^{\prime}(t)=\lambda(-1)^{k} \alpha_{k} w_{k}(t), \quad t \in(0, \omega), \quad \int_{0}^{\omega} w_{k}(s) d s=0, \quad w_{k}(0)=w_{k}(\omega), \quad k=1,2 .
$$

Отсюда следует, что вектор-функции $w_{1}(t)$ и $w_{2}(t)$ тождественно равны нулю.

Из равенств (8) и (10) вытекает, что

$$
\gamma_{\infty}\left(\Phi_{1}\right)=\gamma_{\infty}\left(\Psi_{0}\right)
$$

Векторное поле $\Psi_{0}$ состоит из разности единичного и конечномерного операторов. Поэтому, согласно свойству векторных полей [2], для вычисления вращения $\gamma_{\infty}\left(\Psi_{0}\right)$ достаточно рассмотреть проекцию $\Psi_{0}$ на $\mathbb{C}^{n_{1}} \times \mathbb{C}^{n_{2}}$ :

$$
\gamma_{\infty}\left(\Psi_{0}\right)=\gamma_{\infty}\left(\widetilde{\Psi}_{0}\right)
$$

где

$$
\begin{gathered}
\widetilde{\Psi}_{0}\left(z_{1}, z_{2}\right)=\Psi_{0}\left(z_{1}, z_{2}\right), \quad\left(z_{1}, z_{2}\right) \in \mathbb{C}^{n_{1}} \times \mathbb{C}^{n_{2}} \\
\widetilde{\Psi}_{0}\left(z_{1}, z_{2}\right)=\left(\begin{array}{c}
\omega \alpha_{1} z_{1} \\
-\omega \alpha_{2} z_{2}
\end{array}\right)=\left(\begin{array}{cc}
\omega \alpha_{1} & 0 \\
0 & -\omega \alpha_{2}
\end{array}\right)\left(\begin{array}{l}
z_{1} \\
z_{2}
\end{array}\right) .
\end{gathered}
$$

Для линейного отображения $\widetilde{\Psi}_{0}: \mathbb{C}^{n_{1}} \times \mathbb{C}^{n_{2}} \mapsto \mathbb{C}^{n_{1}} \times \mathbb{C}^{n_{2}}$ его вращение, как известно, равно знаку определителя матрицы отображения [2]:

$$
\gamma_{\infty}\left(\widetilde{\Psi}_{0}\right)=\operatorname{sign}\left(\begin{array}{cc}
\omega \alpha_{1} & 0 \\
0 & -\omega \alpha_{2}
\end{array}\right)=(-1)^{2 n_{2}}=1
$$

Из равенств (8), (11), (12) и (13) следует (9).

Теорема 2 доказана.

5. Существование ограниченного решения. Докажем теорему 3 , приближая правые части системы уравнений (1) их периодическими срезками с возрастающими периодами.

Рассмотрим системы уравнений

$$
v_{k}^{\prime}=F_{k m}\left(t, v_{1}, v_{2}\right), \quad v_{k} \in \mathbb{C}^{n_{k}}, \quad|t|<m+1, \quad k=1,2, \quad m=1,2, \ldots,
$$

где

$$
\begin{gathered}
F_{k m}\left(t, v_{1}, v_{2}\right)=\eta_{m}(t) F_{k}\left(t, v_{1}, v_{2}\right)+\left(1-\eta_{m}(t)\right)(-1)^{k} \alpha_{k} v_{k}, \quad k=1,2, \\
\eta_{m}(t) \in C(R), \quad 0 \leqslant \eta_{m}(t) \leqslant 1, \\
\eta_{m}(t)=1 \text { при }|t| \leqslant m, \quad \eta_{m}(t)=0 \text { при }|t| \geqslant m+1 .
\end{gathered}
$$

При каждом $m$ правые части системы уравнений (14) периодически продолжим по $t$ с периодом $\omega_{m}=2(m+1)$. Легко проверить, что при каждом $m$ правые части системы уравнений (14) удовлетворяют условиям теоремы 2 . Согласно теореме 2 , при каждом $m$ существует $\omega_{m}$-периодическое решение $\left(v_{1 m}(t), v_{2 m}(t)\right)$ системы уравнений $(14)$. Для последовательности $\left(v_{1 m}(t), v_{2 m}(t)\right)$, $m=1,2, \ldots$, в силу теоремы 1 имеет место оценка

$$
\left\|v_{1 m}\right\|_{1}^{2}+\left\|v_{2 m}\right\|_{2}^{2} \leqslant M, \quad m=1,2, \ldots
$$

Из этой оценки в силу систем уравнений (14) вытекает ограниченность последовательности производных $\left(v_{1 m}^{\prime}(t), v_{2 m}^{\prime}(t)\right), m=1,2, \ldots$ Поэтому можно выделить подпоследовательность $\left(v_{1 m_{j}}(t), v_{2 m_{j}}(t)\right), j=1,2, \ldots$, которая равномерно сходится на каждом конечном отрезке. В системе уравнений (14), переходя к пределу вдоль данной подпоследовательности, получим ограниченное решение системы уравнений (1).

Теорема 3 доказана. 
6. Примеры. Рассмотрим примеры систем нелинейных обыкновенных дифференциальных уравнений, к которым можно применить теоремы 2 и 3.

В качестве первого примера рассмотрим систему уравнений

$$
v_{k}^{\prime}=A_{k}\left(t, v_{1}, v_{2}\right) v_{k}+f_{k}\left(t, v_{1}, v_{2}\right), \quad v_{k} \in \mathbb{C}^{n_{k}}, \quad t \in \mathbb{R} .
$$

Здесь отображения $A_{k}: \mathbb{R} \times \mathbb{C}^{n_{1}} \times \mathbb{C}^{n_{2}} \mapsto L\left(\mathbb{C}^{n_{k}}\right), k=1,2$, где $L\left(\mathbb{C}^{n_{k}}\right)$ - пространство квадратных матриц порядка $n_{k}$, и отображения $f_{k}: \mathbb{R} \times \mathbb{C}^{n_{1}} \times \mathbb{C}^{n_{2}} \mapsto \mathbb{C}^{n_{k}}, k=1,2$, непрерывны и при всех $t \in \mathbb{R}, z_{1} \in \mathbb{C}^{n_{1}}, z_{2} \in \mathbb{C}^{n_{2}}$ удовлетворяют условиям:

$$
\begin{gathered}
(-1)^{k}<\left(A_{k}+A_{k}^{*}\right)\left(t, z_{1}, z_{2}\right) z_{k}, z_{k}>_{k} \geqslant 2 a_{k}\left|z_{k}\right|_{k}^{2}, \quad k=1,2, \\
\left|f_{k}\left(t, z_{1}, z_{2}\right)\right|_{k} \leqslant b_{k}\left(\left|z_{1}\right|_{1}+\left|z_{2}\right|_{2}\right)+c_{k}, \quad k=1,2,
\end{gathered}
$$

где $a_{k}, b_{k}, c_{k}, k=1,2$ - вещественные неотрицательные числа.

Следствие 1. Система уравнений (15) имеет хотя бы одно ограниченное решение, если выполнены условия (16), (17) и

$$
a_{k}>1,5 b_{k}, \quad k=1,2, \quad\left(a_{1}-1,5 b_{1}\right)\left(a_{2}-1,5 b_{2}\right)>0,25 b_{1} b_{2} .
$$

Проверим, что отображения

$$
F_{k}\left(t, z_{1}, z_{2}\right)=A_{k}\left(t, z_{1}, z_{2}\right) z_{k}+f_{k}\left(t, z_{1}, z_{2}\right), \quad k=1,2,
$$

удовлетворяют условиям (2)-(4). Выберем $\varepsilon>0$ из условий

$$
a_{k}>(1,5+\varepsilon) b_{k}, \quad k=1,2, \quad\left(a_{1}-(1,5+\varepsilon) b_{1}\right)\left(a_{2}-(1,5+\varepsilon) b_{2}\right)>0,25 b_{1} b_{2} .
$$

Положим

$$
\alpha_{1}=\left(a_{1}-(1,5+\varepsilon) b_{1}\right), \quad \beta_{1}=0,5 b_{1}, \quad \gamma_{1}=\max _{\lambda \geqslant 0}\left(c_{1} \lambda-\varepsilon b_{1} \lambda^{2}\right),
$$

и проверим выполнимость условия (2):

$$
\begin{gathered}
\operatorname{Re}\left\langle A_{1}\left(t, z_{1}, z_{2}\right) z_{1}+f_{1}\left(t, z_{1}, z_{2}\right), z_{1}\right\rangle_{1}= \\
=0,5\left\langle\left(A_{1}+A_{1}^{*}\right)\left(t, z_{1}, z_{2}\right) z_{1}, z_{1}\right\rangle_{1}+\operatorname{Re}\left\langle f_{1}\left(t, z_{1}, z_{2}\right), z_{1}\right\rangle_{1} \leqslant \\
\leqslant-a_{1}\left|z_{1}\right|_{1}^{2}+\left.\left|z_{1}\right|\right|_{1}\left(b_{1}\left|z_{1}\right|_{1}+b_{1}\left|z_{2}\right|_{2}+c_{1}\right)=\left(b_{1}-a_{1}\right)\left|z_{1}\right|_{1}^{2}+c_{1}\left|z_{1}\right|_{1}+b_{1}\left|z_{1}\right|_{1}\left|z_{2}\right|_{2} \leqslant \\
\quad \leqslant\left(b_{1}-a_{1}\right)\left|z_{1}\right|_{1}^{2}+c_{1}\left|z_{1}\right|_{1}+0,5 b_{1}\left|z_{1}\right|_{1}^{2}+0,5 b_{1}\left|z_{2}\right|_{2}^{2}= \\
=-\left(a_{1}-(1,5+\varepsilon) b_{1}\right)\left|z_{1}\right|_{1}^{2}+0,5 b_{1}\left|z_{2}\right|_{2}^{2}+\left(c_{1}\left|z_{1}\right|_{1}-\varepsilon b_{1}\left|z_{1}\right|_{1}^{2}\right) \leqslant-\alpha_{1}\left|z_{1}\right|_{1}^{2}+\beta_{1}\left|z_{2}\right|_{2}^{2}+\gamma_{1} .
\end{gathered}
$$

Аналогичным образом, полагая

$$
\alpha_{2}=\left(a_{2}-(1,5+\varepsilon) b_{2}\right), \quad \beta_{1}=0,5 b_{2}, \quad \gamma_{2}=-\min _{\lambda \geqslant 0}\left(\varepsilon b_{2} \lambda^{2}-c_{2} \lambda\right),
$$

проверим выполнимость условия (3):

$$
\begin{gathered}
\operatorname{Re}\left\langle A_{2}\left(t, z_{1}, z_{2}\right) z_{2}+f_{2}\left(t, z_{1}, z_{2}\right), z_{2}\right\rangle_{2}= \\
=0,5\left\langle\left(A_{2}+A_{2}^{*}\right)\left(t, z_{1}, z_{2}\right) z_{2}, z_{2}\right\rangle_{2}+\operatorname{Re}\left\langle f_{2}\left(t, z_{1}, z_{2}\right), z_{2}\right\rangle_{2} \geqslant \\
\geqslant a_{2}\left|z_{2}\right|_{2}^{2}-\left|z_{2}\right|_{2}\left(b_{2}\left|z_{1}\right|_{1}+b_{2}\left|z_{2}\right|_{2}+c_{2}\right)=\left(a_{2}-b_{2}\right)\left|z_{2}\right|_{2}^{2}-c_{2}\left|z_{2}\right|_{2}-\left.b_{2}\left|z_{1}\right|\right|_{1}\left|z_{2}\right|_{2} \geqslant \\
\geqslant\left(a_{2}-b_{2}\right)\left|z_{2}\right|_{2}^{2}-\left.c_{2}\left|z_{2}\right|\right|_{2}-0,5 b_{2}\left|z_{1}\right|_{1}^{2}-0,5 b_{2}\left|z_{2}\right|_{2}^{2}= \\
=\left(a_{2}-(1,5+\varepsilon) b_{2}\right)\left|z_{2}\right|_{2}^{2}-0,5 b_{2}\left|z_{1}\right|_{1}^{2}+\left(\varepsilon b_{2}\left|z_{2}\right|_{2}^{2}-c_{2}\left|z_{2}\right|_{2}\right) \geqslant \alpha_{2}\left|z_{2}\right|_{2}^{2}-\beta_{2}\left|z_{1}\right|_{2}^{2}-\gamma_{2} .
\end{gathered}
$$

Очевидно, условие (4) выполнено. Таким образом, правые части системы уравнений (15) удовлетворяют условиям теоремы 3 , и следствие 1 действительно верно.

В качестве второго примера рассмотрим систему уравнений

$$
x^{\prime}=|x|^{p} x+f(t, x, y), \quad y^{\prime}=-|y|^{q} y+g(t, x, y), \quad t, x, y \in \mathbb{R} .
$$

Здесь $p \geqslant 0, q \geqslant 0$, функции $f(t, x, y)$ и $g(t, x, y)$ непрерывны по совокупности переменных, $\omega$ периодичны по $t$ и удовлетворяют условиям

$$
|f(t, x, y)| \leqslant f_{1}(|x|+|y|)+f_{2}, \quad|g(t, x, y)| \leqslant g_{1}(|x|+|y|)+g_{2},
$$


где $f_{1}, f_{2}, g_{1}, g_{2}$ - неотрицательные числа, на них наложим некоторые условия.

Проверим, что функции

$$
F_{1}(t, x, y)=|x|^{p} x+f(t, x, y), \quad F_{2}(t, x, y)=-|y|^{q} y+g(t, x, y)
$$

удовлетворяют условиям (2) и (3). Для функции $F_{1}(t, x, y)$, учитывая первое из условий $(20)$, имеем:

$$
x F(t, x, y)=|x|^{p} x^{2}+x f(t, x, y) \geqslant|x|^{p} x^{2}-|x|\left(f_{1}(|x|+|y|)+f_{2}\right) \geqslant\left(|x|^{p}-f_{1}\right) x^{2}-f_{1}|x||y|-f_{2}|x| .
$$

Покажем, что существуют положительные числа $\alpha_{1}, \beta_{1}$ и $\gamma_{1}$ такие, что $\alpha_{1}>\beta_{1}$ и при всех $t, x, y \in \mathbb{R}$ выполняется неравенство

$$
\left(|x|^{p}-f_{1}\right) x^{2}-f_{1}|x||y|-f_{2}|x| \geqslant \alpha_{1} x^{2}-\beta_{1} y^{2}-\gamma_{1} .
$$

Действительно, левую часть оценим снизу следующим образом:

$$
\left(|x|^{p}-f_{1}\right) x^{2}-f_{1}|x||y|-f_{2}|x| \geqslant\left(|x|^{p}-f_{1}\right) x^{2}-0,5 f_{1}\left(x^{2}+y^{2}\right)-f_{2}|x| .
$$

Подберем $\alpha_{1}, \beta_{1}$ и $\gamma_{1}$ так, чтобы выполнялось неравенство

$$
\left(|x|^{p}-f_{1}\right) x^{2}-0,5 f_{1}\left(x^{2}+y^{2}\right)-f_{2}|x| \geqslant \alpha_{1} x^{2}-\beta_{1} y^{2}-\gamma_{1},
$$

Рассмотрим два случая: $p=0$ и $p>0$. В первом случае неравенство принимает вид

$$
\left(1-1,5 f_{1}-\alpha_{1}\right) x^{2}+\left(\beta_{1}-0,5 f_{1}\right) y^{2}-f_{2}|x| \geqslant-\gamma_{1} .
$$

Считая $0 \leqslant f_{1}<0,5$, возьмем

$$
\beta_{1}=0,5 f_{1}, \quad \beta_{1}<\alpha_{1}<1-1,5 f_{1}, \quad \gamma_{1}=-\min _{\lambda \geqslant 0}\left(\left(1-1,5 f_{1}-\alpha_{1}\right) \lambda^{2}-f_{2} \lambda\right) .
$$

Во втором случае, когда $p>0$, полагая $\beta_{1}=0,5 f_{1}$ и $\alpha_{1}>0,5 f_{1}$, имеем:

$$
\left(|x|^{p}-\alpha_{1}-1,5 f_{1}\right) x^{2}-f_{2}|x| \geqslant-\gamma_{1},
$$

где

Таким образом, функция

$$
\gamma_{1}=-\min _{\lambda \geqslant 0}\left(\left(\lambda^{p}-\alpha_{1}-1,5 f_{1}\right) \lambda^{2}-f_{2} \lambda\right)
$$

$$
F_{1}(t, x, y)=|x|^{p} x+f(t, x, y)
$$

удовлетворяет условию (2) при некоторых $\alpha_{1}>\beta_{1} \geqslant 0$ и $\gamma_{1} \geqslant 0$, если либо $p=0$ и $f_{1}<0,5$, либо $p>0$. Аналогичным образом можно проверить, что функция

$$
F_{2}(t, x, y)=-|y|^{q} y+g(t, x, y)
$$

удовлетворяет условию (3) при некоторых $\alpha_{2}>\beta_{2} \geqslant 0$ и $\gamma_{2} \geqslant 0$, если либо $q=0$ и $g_{1}<0,5$, либо $q>0$. Отсюда в силу теорем 1 и 2 вытекает

Следствие 2. Если функиии $f(t, x, y)$ u $g(t, x, y)$ непрерывны по совокупности переменных, $\omega$-периодичны по $t$, удовлетворяют условиям (20) и при $p=0$ выполнено неравенство $f_{1}<$ 0,5 , а при $q=0$-неравенство $g_{1}<0,5$, то ограниченные решения системы уравнений (19) допускают априорную оченку, и среди них есть хотл бы одно $\omega$-периодическое решение.

В качестве третьего примера рассмотрим нелинейное уравнение второго порядка следующего вида

$$
x^{\prime \prime}-p^{2}(t, x) x=q\left(t, x, x^{\prime}\right), \quad t, x \in \mathbb{R},
$$

где функции $p(t, \xi)$ и $q(t, \xi, \eta)$ непрерывны по совокупности переменных. В этом уравнении произведем замену

Тогда для $y(t)$ получим уравнение

$$
x(t)=y(\mu t), \quad \mu>0 .
$$

$$
y^{\prime \prime}-\mu^{-2} p^{2}\left(\mu^{-1} t, y\right) y=\mu^{-2} q\left(\mu^{-1} t, y, \mu y^{\prime}\right) .
$$

Данное уравнение представим в следующем виде:

$$
\left(\frac{d}{d t}+\mu^{-1} p\right)\left(\frac{d}{d t}-\mu^{-1} p\right) y=-\mu^{-1} y\left(\mu^{-1} \frac{\partial p}{\partial t}+\frac{\partial p}{\partial y^{\prime}} y^{\prime}\right)+\mu^{-2} q\left(\mu^{-1} t, y, \mu y^{\prime}\right) .
$$


Вводя обозначения $z_{1}=y, z_{2}=y^{\prime}-\mu^{-1} p\left(\mu^{-1} t, y\right) y$,

$$
\begin{aligned}
g_{\mu}\left(t, z_{1}, z_{2}\right)=\mu^{-2} q\left(\mu^{-1} t, z_{1}, \mu z_{2}+p\left(\mu^{-1} t, z_{1}\right) z_{1}\right)- & \\
-\mu^{-1} z_{1} & {\left[\mu^{-1} \frac{\partial p}{\partial t}\left(\mu^{-1} t, z_{1}\right)+\frac{\partial p}{\partial z_{1}}\left(\mu^{-1} t, z_{1}\right)\left(z_{2}+\mu^{-1} p\left(\mu^{-1} t, z_{1}\right) z_{1}\right)\right], }
\end{aligned}
$$

относительно $z_{1}(t)$ и $z_{2}(t)$ получим систему уравнений

$$
\left\{\begin{array}{l}
z_{1}^{\prime}=\mu^{-1} p\left(\mu^{-1} t, z_{1}\right) z_{1}+z_{2} \\
z_{2}^{\prime}=-\mu^{-1} p\left(\mu^{-1} t, z_{1}\right) z_{2}+g_{\mu}\left(t, z_{1}, z_{2}\right) .
\end{array}\right.
$$

Предположим, что существуют $\mu>0, p_{0}>1,0 \leqslant g_{0}<0,5 p_{0}, g_{1} \geqslant 0$ такие, что при всех $t, z_{1}, z_{2} \in \mathbb{R}$ выполняются условия

$$
\begin{gathered}
\mu^{-1} p\left(\mu^{-1} t, z_{1}\right) \geqslant p_{0}, \\
\left|g_{\mu}\left(t, z_{1}, z_{2}\right)\right| \leqslant g_{0}\left(\left|z_{2}\right|+\left|z_{2}\right|\right)+g_{1} .
\end{gathered}
$$

Проверим, что правые части системы уравнений (22) удовлетворяют условиям (2), (3):

$$
\begin{gathered}
\left(\mu^{-1} p\left(\mu^{-1} t, z_{1}\right) z_{1}+z_{2}\right) z_{1} \geqslant p_{0} z_{1}^{2}-0,5 z_{1}^{2}-0,5 z_{2}^{2}=\left(p_{0}-0,5\right) z_{1}^{2}-0,5 z_{2}^{2}, \\
\alpha_{1}=p_{0}-0,5, \quad \beta_{1}=0,5, \quad \gamma_{1}=0 ; \\
\left(-\mu^{-1} p\left(\mu^{-1} t, z_{1}\right) z_{2}+g_{\mu}\left(t, z_{1}, z_{2}\right)\right) z_{2} \leqslant-p_{0} z_{2}^{2}+g_{0}\left(\left|z_{1}\right|+\left|z_{2}\right|\right)\left|z_{2}\right|+g_{1}\left|z_{2}\right| \leqslant \\
\leqslant-\left(p_{0}-g_{0}\right) z_{2}^{2}+0,5 g_{0}\left(z_{1}^{2}+z_{2}^{2}\right)+g_{1}\left|z_{2}\right|=-\left(p_{0}-1,5 g_{0}\right) z_{2}^{2}+0,5 g_{0} z_{1}^{2}+g_{1}\left|z_{2}\right|= \\
=-\left(p_{0}-(1,5+\varepsilon) g_{0}\right) z_{2}^{2}+0,5 g_{0} z_{1}^{2}+g_{1}\left|z_{2}\right|-\varepsilon g_{0} z_{2}^{2} .
\end{gathered}
$$

Выберем $\varepsilon$ так, чтобы $p_{0}>(2+\varepsilon) g_{0}$. Тогда, полагая

$$
\alpha_{2}=p_{0}-(1,5+\varepsilon) g_{0}, \quad \beta_{2}=0,5 g_{0}, \quad \gamma_{2}=\max _{\lambda \geqslant 0}\left(g_{1} \lambda-\varepsilon g_{0} \lambda^{2}\right)
$$

имеем:

$$
\left(-\mu^{-1} p\left(\mu^{-1} t, z_{1}\right) z_{2}+g_{\mu}\left(t, z_{1}, z_{2}\right)\right) z_{2} \leqslant-\alpha_{2} z_{2}^{2}+\beta_{2} z_{1}^{2}+\gamma_{2} .
$$

Заметим, что $\beta_{1}<\alpha_{1}$ и $\beta_{2}<\alpha_{2}$, т.е. условие (4) выполнено. Отсюда в силу теорем 1,2 и 3 вытекает следующее утверждение.

Следствие 3. Если выполнены условия (23), (24), где $\mu>0, p_{0}>1,0 \leqslant g_{0}<0,5 p_{0}, g_{1} \geqslant 0$, и выполнено условие

$$
\sup _{t \in \mathbb{R}} p(t, \xi)<\infty
$$

то для любого решения $x(t)$ уравнения (21), определенного и ограниченного на всей числовой оси вместе со своей производной $x^{\prime}(t)$, имеет место априорная оценка

$$
\sup _{t \in \mathbb{R}}|x(t)|+\sup _{t \in \mathbb{R}}\left|x^{\prime}(t)\right|<R .
$$

Если к тому же функиии $p(t, \xi)$ и $q(t, \xi, \eta) \omega$-периодичны по $t$, то существует $\omega$-периодическое решение уравнения (21).

Следствие 3 можно применить к уравнению

$$
x^{\prime \prime}-4\left(1+e^{x}\right)^{2} x=2 x e^{x} x^{\prime}+q_{0}\left(t, x, x^{\prime}\right),
$$

где функция $q_{0}(t, \xi, \eta)$ непрерывна по совокупности переменных и ограничена

$$
\sup _{(t, \xi, \eta) \in \mathbb{R}^{3}}\left|q_{0}(t, \xi, \eta)\right|<\infty .
$$


Полагая $\mu=1, p_{0}=2, g_{0}=0$, имеем:

$$
\begin{gathered}
g_{\mu}\left(t, z_{1}, z_{2}\right)=2 z_{1} e^{z_{1}}\left(z_{2}+2\left(1+e^{z_{1}}\right) z_{1}\right)+ \\
q_{0}\left(t, z_{1}, z_{2}+2\left(1+e^{z_{1}}\right) z_{1}\right)- \\
-2 z_{1} e^{z_{1}}\left(z_{2}+2\left(1+e^{z_{1}}\right) z_{1}\right)=q_{0}\left(t, z_{1}, z_{2}+2\left(1+e^{z_{1}}\right) z_{1}\right), \\
\left|g_{\mu}\left(t, z_{1}, z_{2}\right)\right| \leqslant \sup _{(t, \xi, \eta) \in \mathbb{R}^{3}}\left|q_{0}(t, \xi, \eta)\right|=g_{1} .
\end{gathered}
$$

Следовательно, для любого решения уравнение (27) имеет место априорная оценка (26). А если функция $q_{0}(t, \xi, \eta)$ периодична по переменной $t$ с периодом $\omega$, то существует $\omega$-периодическое решение уравнения (27).

\section{СПИСОК ЛИТЕРАТУРЫ}

1. Красносельский M. А. Оператор сдвига по траекториям дифференциальных уравнений. - М.: Наука, 1966.

2. Красносельский М. А., Забрейко П. П. Геометрические методы нелинейного анализа. - М.: Наука, 1975.

3. Мухамадиев Э. К теории периодических решений систем обыкновенных дифференциальных уравнений // Докл. АН СССР. - 1970. - 194, № 11. - С. 510-513.

4. Мухамадиев Э. К теории ограниченных решений обыкновенных дифференциальных уравнений// Диффер. уравн. - 1974. - 10, № 4. - С. 635-646.

5. Мухамадиев Э. Исследования по теории периодических и ограниченных решений дифференциальных уравнений// Мат. заметки. - 1981. - 30, № 3. - С. 713-722.

6. Мухамадиев Э., Наимов А. Н., Сатторов А. Х. Об ограниченных решениях одного класса гиперболических уравнений на плоскости// Диффер. уравн. - 2016. - 52, № 1. - С. 86-93.

7. Мухамадиев Э., Наимов А. Н., Сатторов А. Х. Аналог теоремы Боля для одного класса линейных дифференциальных уравнений в частных производных// Уфим. мат. ж. -2017 . - 9, № 1. - С. 75-88.

Кобилзода Мирзоодили Мирзомалик

Таджикский национальный университет, Душанбе, Таджикистан

E-mail: kobilzoda94@mail.ru

Наимов Алижон Набиджанович

Вологодский государственный университет

E-mail: nan67@rambler.ru 